\title{
Proof of the identity between the depletion layer thickness and half the average span
} for an arbitrary polymer chain

\author{
Wang, Yanwei; Peters, Günther H.J.; Hansen, Flemming Yssing; Hassager, Ole
}

Published in:

Journal of Chemical Physics

Link to article, DOI:

$10.1063 / 1.2970935$

Publication date:

2008

Document Version

Publisher's PDF, also known as Version of record

Link back to DTU Orbit

Citation (APA):

Wang, Y., Peters, G. H. J., Hansen, F. Y., \& Hassager, O. (2008). Proof of the identity between the depletion layer thickness and half the average span for an arbitrary polymer chain. Journal of Chemical Physics, 129(7), [074904]. https://doi.org/10.1063/1.2970935

\section{General rights}

Copyright and moral rights for the publications made accessible in the public portal are retained by the authors and/or other copyright owners and it is a condition of accessing publications that users recognise and abide by the legal requirements associated with these rights.

- Users may download and print one copy of any publication from the public portal for the purpose of private study or research.

- You may not further distribute the material or use it for any profit-making activity or commercial gain

- You may freely distribute the URL identifying the publication in the public portal 


\title{
Proof of the identity between the depletion layer thickness and half the average span for an arbitrary polymer chain
}

\author{
Yanwei Wang, ${ }^{1, a)}$ Flemming Y. Hansen, ${ }^{2, b)}$ Günther H. Peters, ${ }^{3, c)}$ and Ole Hassager ${ }^{1, d)}$ \\ ${ }^{1}$ Danish Polymer Center, Department of Chemical and Biochemical Engineering, Technical \\ University of Denmark, DK-2800 Kgs. Lyngby, Denmark \\ ${ }^{2}$ Department of Chemistry, Technical University of Denmark, DK-2800 Kgs. Lyngby, Denmark \\ ${ }^{3}$ MEMPHYS-Center for Biomembrane Physics, Department of Chemistry, Technical University \\ of Denmark, DK-2800 Kgs. Lyngby, Denmark
}

(Received 3 June 2008; accepted 23 July 2008; published online 21 August 2008)

\begin{abstract}
The confinement analysis from bulk structure (CABS) approach [Y. Wang et al., J. Chem. Phys. 128, 124904 (2008)] is extended to determine the depletion profiles of dilute polymer solutions confined to a slit or near an inert wall. We show that the entire spatial density distributions of any reference point in the polymer chain (such as the center of mass, middle segment, and end segments) can be computed as a function of the confinement size solely based on a single sampling of the configuration space of a polymer chain in bulk. Through a simple analysis based on the CABS approach in the case of a single wall, we prove rigorously that (i) the depletion layer thickness $\delta$ is the same no matter which reference point is used to describe the depletion profile and (ii) the value of $\delta$ equals half the average span (the mean projection onto a line) of the macromolecule in free solution. Both results hold not only for ideal polymers, as has been noticed before, but also for polymers regardless of details in molecular architecture and configuration statistics. (c) 2008 American Institute of Physics. [DOI: 10.1063/1.2970935]
\end{abstract}

\section{INTRODUCTION}

The properties of spatially confined macromolecules are both of fundamental interest in polymer physics ${ }^{1-6}$ and of practical importance in a variety of applications including chromatographic fractionation of polymers, ${ }^{7-12}$ polymer improved oil recovery, ${ }^{13,14}$ and applications of polymers in colloidal suspensions. ${ }^{15-17}$ For example, the entropic depletion of polymers near inert hard surfaces due to steric exclusion effects effectively leads to an attraction force (the so-called depletion interaction $)^{18-20}$ that may drive aggregation of colloidal particles, ${ }^{21-23}$ bacteria, ${ }^{24}$ and cells. ${ }^{25,26}$ To provide an understanding of the depletion interaction, it is important to determine the depletion profiles of polymers near confining surfaces. ${ }^{27}$

In a recent effort of determining the equilibrium partitioning of polymers between a confining geometry and a dilute bulk solution, we presented the confinement analysis from bulk structure (CABS) method. ${ }^{28}$ By using snapshots of molecular configurations of an isolated polymer chain in free space to estimate the effect of spatial confinement, CABS has the superior property of obtaining the equilibrium partition coefficient $K_{0}$ as a function of the confinement size, solely on the basis of a single sampling of the molecular configuration space. However, $K_{0}$ describes only the mean concentration of polymers inside the confining geometry relative to the bulk concentration and not any spatial anisotropy near confining surfaces.

\footnotetext{
${ }^{a)}$ Electronic mail: wyw@kt.dtu.dk.

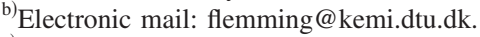

${ }^{c}$ Electronic mail: ghp@kemi.dtu.dk.

d)Electronic mail: oh@kt.dtu.dk.
}

In the present work, the CABS method is extended to determine the depletion profiles of polymers near confining surfaces. We focus on the equilibrium distribution of polymers in dilute solution near impenetrable but otherwise passive plane boundaries, in which case, the computational efficiency of the CABS method is preserved, i.e., the entire spatial depletion profiles can be computed as a function of the confinement size on the basis of only a single sampling of the configuration space of a polymer chain in bulk. Note that this classical problem was first studied by Asakura and Oosawa in $1954 .{ }^{18}$ It has long been regarded as a wellunderstood problem, ${ }^{29,30}$ since it can be studied by a variety of known procedures, including methodology in analogy with the diffusion problem in the presence of absorbing boundaries, ${ }^{31-38}$ Monte Carlo calculations, ${ }^{39-41}$ and novel mesoscopic simulation techniques such as Brownian dynamics (BD), ${ }^{42,43}$ Lattice Boltzmann, ${ }^{44,45}$ and dissipative particle dynamics (DPD). ${ }^{46,47}$ It has been found that the theoretically calculated depletion profiles agree, in general, with the available experimental data. ${ }^{48,49}$ They are concentration dependent ${ }^{50-53}$ and coupled at flow conditions to the hydrodynamic interaction between polymers and the confining geometry. ${ }^{54}$ Even in the case of no flow and in the dilute solution limit, the depletion profiles are still influenced by, e.g., the molecular architecture and configuration statistics (such as Gaussian chain and excluded volume chain) of the polymer chain. Besides, depending on the reference point (such as the center of mass, endpoints, and midpoint in the polymer chain) used to describe the polymer depletion, the resulting density distributions are different. ${ }^{36,38,51}$ Attempts have been made to identify possible universality in the depletion profiles. Notable among these efforts is the use of the 
depletion layer thickness $\delta$, which describes the range of a step function such that it excludes exactly the same amount of polymers as the true density profile. ${ }^{51}$ For instance, the mean segment density profile of a polymer chain in the vicinity of a single wall can be empirically expressed by the square of a hyperbolic tangent: ${ }^{55,56}$ i.e., $\dot{\bar{\rho}}^{(1)}(x) / \rho_{0}$ $\approx \tanh ^{2}(x / \delta)$, where $\bar{\rho}^{(1)}(x)=\Sigma_{1}^{N} \rho_{i}^{(1)}(x) / N$ is the mean segment density profile for a chain of $N$ segments with $\rho_{i}^{(1)}(x)$ as the density profile of the $i$ th chain segment located at a distance $x$ to the wall. $\rho_{0}$ is the uniform density far from the wall, where $x \rightarrow+\infty$. The subscript in symbol $\rho_{i}^{(1)}(x)$ indicates that the $i$ th segment is used as the reference point in the description of the depletion profile. The superscript is (1) for the case of a single wall and (2) for a slit confining geometry. As shown, e.g., in Refs. 38 and 57, this empirical relation describes well the analytical results for linear and symmetric star ideal chains, numerical results from mean-field lattice calculations, ${ }^{58}$ and simulation results for excluded volume chains. ${ }^{51}$ Recently, it was noticed by Fedosov et al. ${ }^{47}$ that the center of mass (com) depletion profiles for linear bead-spring polymers of different total number of beads collapse onto "an almost common curve," when the depletion thickness $\delta$ was used to normalize the distance to the wall as opposed to the commonly used radius of gyration $R_{g}$ of the polymer chain in bulk. Although a fundamental proof of the existence of those empirical universal relations is still lacking, the significance of the depletion layer thickness $\delta$ is nevertheless underlined.

When the depletion layer thickness $\delta$ is calculated in theoretical studies of polymer depletion near a single wall, it has been noticed for a linear random-flight chain in the continuous limit (the so-called ideal chain) that irrespective of whether one uses the com, the endpoint, or the midpoint density distributions, the resulting depletion layer thickness is the same, i.e.,

$$
\begin{aligned}
\delta=\int_{0}^{+\infty}\left(1-\frac{\rho_{\mathrm{com}}^{(1)}(x)}{\rho_{0}}\right) d x & =\int_{0}^{+\infty}\left(1-\frac{\bar{\rho}^{(1)}(x)}{\rho_{0}}\right) d x \\
& =\int_{0}^{+\infty}\left(1-\frac{\rho_{i}^{(1)}(x)}{\rho_{0}}\right) d x,
\end{aligned}
$$

and the value of $\delta$ equals $2 R_{g} / \sqrt{\pi}$, which happens to be half the average span of a linear ideal chain. ${ }^{34-38,51,58-64}$

The span of a polymer spatial configuration in a given direction $\boldsymbol{e}_{k}$ is defined as the minimum distance between parallel planes normal to $\boldsymbol{e}_{k}$ that entirely contain the chain. That is to say, for a random walker, the span in the $x$ direction at a fixed time is the maximum $x$ displacement of the random walker minus the minimum $x$ displacement. The average span of a polymer chain is a measure of the molecular size that has been studied frequently ${ }^{65-76}$ since the pioneering work by Daniels ${ }^{77}$ and Kuhn ${ }^{78,79}$ in the 1940s. As noted by Casassa, ${ }^{36}$ this quantity has been called differently, such as the "extent,"77 the "span," the "mean maximal projection," 67 or, more descriptively, the "mean projection onto a line." 66 In Ref. 28 , we denoted it by $\langle\sigma\rangle$ and introduced the steric exclusion radius $R_{s}$ of a polymer chain in bulk as half the average span: i.e., $R_{s}=\langle\sigma\rangle / 2$,

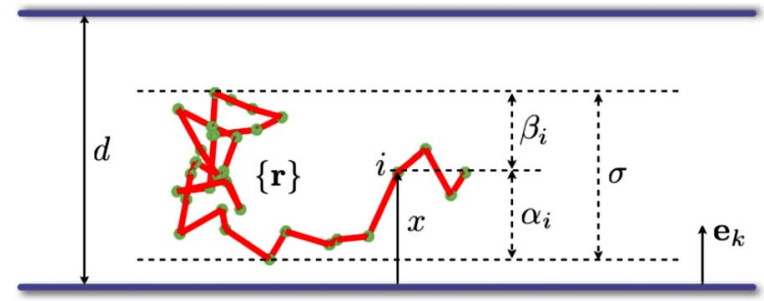

FIG. 1. (Color online) Schematic illustration of a polymer chain in a slit confinement. The confinement size is denoted by $d$, and the orientation of the slit is denoted by $\mathbf{e}_{k}$, which is a unit vector normal to the slit plane. $\sigma$ is the span in the $\mathbf{e}_{k}$ direction of the polymer chain with configuration $\{\mathbf{r}\}$, which is divided into two parts, $\alpha_{i}$ and $\beta_{i}$, by the projection point of bead $i$ located at distance $x$ from the slit plane at $x=0$.

where $\langle\cdots\rangle$ refers to an ensemble average over the configurational degrees of freedom of the chain. $R_{s}$ is called the steric exclusion radius because it is deduced directly from a study of the steric exclusion effect introduced by confining geometries, and it is a more relevant polymer size parameter than the radius of gyration $R_{g}$ in size exclusion chromatography. ${ }^{28,35}$ As discussed by Casassa, ${ }^{37,38}$ the identity relation

$$
\delta=R_{s}
$$

between the depletion layer thickness $\delta$ and half the average span of a polymer chain, i.e., the steric exclusion radius $R_{s}$, holds not only for linear ideal chains but also for rigid molecules $^{8}$ and ideal polymers of nonlinear architecture, such as an $f$-arm symmetric star molecule. ${ }^{38}$ One might speculate that Eqs. (1) and (2) should also hold for Gaussian polymers with finite total number of segments as well as excluded volume chains. However, it has not been strictly proven yet, and a firm proof is still missing.

In this paper, we give a rigorous proof for Eqs. (1) and (2). We show that both equations hold, in fact, for polymers regardless of details in molecular architecture and configuration statistics. This is done in Sec. III. The proof is based on the CABS approach of determining the depletion profiles by analyzing free-space polymer chain statistics as presented in Sec. II. It is straightforward to implement the CABS approach numerically. As an example, we present in Sec. IV simulation results for the depletion profiles of linear Gaussian bead-spring polymers of $N$ beads. Finally, a summary is given in Sec. V.

\section{THE CABS APPROACH}

We first consider the depletion profile of a polymer chain that is confined in a slit of width $d$ (i.e., two infinite parallel planes separated by a distance $d$ ), as shown schematically in Fig. 1. Suppose that the $i$ th segment is placed at distance $x$ from the plane at $x=0$, and both planes of the slit confinement are impenetrable to the polymer but otherwise passive. Based on the procedures outlined in Ref. 28, one can write the fraction of polymer configurations that are not intersected by the confining boundary as 


$$
\frac{\rho_{i}^{(2)}(x, d)}{\rho_{0}}=\left\langle H\left(x-\alpha_{i}\right) H\left(d-\beta_{i}-x\right)\right\rangle,
$$

where $H(x)$ is the Heaviside function, corresponding to the Boltzmann factor of a hard-wall potential between the polymer and the slit plane, ${ }^{20} \alpha_{i}$ and $\beta_{i}$ are the maximum distances (spans) from the $i$ th bead to any other bead in two opposite directions, as denoted by $-\mathbf{e}_{k}$ and $\mathbf{e}_{k}$, respectively. They are given by

$$
\begin{aligned}
& \alpha_{i}=\mathbf{r}_{\mathbf{i}} \cdot \mathbf{e}_{k}-\min \left(\{\mathbf{r}\} \cdot \mathbf{e}_{k}\right), \\
& \beta_{i}=\max \left(\{\mathbf{r}\} \cdot \mathbf{e}_{k}\right)-\mathbf{r}_{\mathbf{i}} \cdot \mathbf{e}_{k},
\end{aligned}
$$

where $\{\mathbf{r}\} \equiv\left\{\mathbf{r}_{1}, \ldots, \mathbf{r}_{i}, \ldots, \mathbf{r}_{N}\right\}$ denotes the collection of monomer position vectors in a polymer chain and $\mathbf{e}_{k}$ is a unit vector normal to the slit plane.

In the case of $d \rightarrow \infty$, Eq. (3) reduces to the result for a polymer chain in the vicinity of the a single wall

$$
\frac{\rho_{i}^{(1)}(x)}{\rho_{0}}=\left\langle H\left(x-\alpha_{i}\right)\right\rangle .
$$

The mean segment density profile $\bar{\rho}^{(1)}(x) / \rho_{0}$ is simply

$$
\frac{\bar{\rho}^{(1)}(x)}{\rho_{0}}=\left\langle\frac{1}{N} \sum_{i=1}^{N} H\left(x-\alpha_{i}\right)\right\rangle .
$$

Similar to Eqs. (4) and (5), the two span components associated with the com (geometric center in the strict sense) of the chain, $\alpha_{\mathrm{com}}$ and $\beta_{\text {com }}$, are introduced in the form

$$
\begin{aligned}
& \alpha_{\mathrm{com}}=\mathbf{r}_{\mathrm{com}} \cdot \mathbf{e}_{k}-\min \left(\{\mathbf{r}\} \cdot \mathbf{e}_{k}\right), \\
& \beta_{\mathrm{com}}=\max \left(\{\mathbf{r}\} \cdot \mathbf{e}_{k}\right)-\mathbf{r}_{\mathrm{com}} \cdot \mathbf{e}_{k},
\end{aligned}
$$

where the position vector of the com of the chain $\mathbf{r}_{\text {com }}$ is given by

$$
\mathbf{r}_{\mathrm{com}}=\frac{1}{N} \sum_{i=1}^{N} \mathbf{r}_{i}
$$

Substituting Eq. (10) into Eqs. (8) and (9) and using the definitions of $\alpha_{i}$ and $\beta_{i}$ in Eqs. (4) and (5), one can show that

$$
\alpha_{\mathrm{com}}=\frac{1}{N} \sum_{i=1}^{N} \alpha_{i}, \quad \beta_{\mathrm{com}}=\frac{1}{N} \sum_{i=1}^{N} \beta_{i} .
$$

By analogy to Eqs. (3) and (6), the density profiles of the com of the chain, $\rho_{\text {com }}^{(2)}(x) / \rho_{0}$ and $\rho_{\text {com }}^{(1)}(x) / \rho_{0}$, can be expressed as

$$
\begin{aligned}
& \frac{\rho_{\mathrm{com}}^{(2)}(x)}{\rho_{0}}=\left\langle H\left(x-\frac{1}{N} \sum_{i=1}^{N} \alpha_{i}\right) H\left(d-\frac{1}{N} \sum_{i=1}^{N} \beta_{i}-x\right)\right\rangle, \\
& \frac{\rho_{\mathrm{com}}^{(1)}(x)}{\rho_{0}}=\left\langle H\left(x-\frac{1}{N} \sum_{i=1}^{N} \alpha_{i}\right)\right\rangle .
\end{aligned}
$$

Note the difference between $\rho_{\mathrm{com}}^{(1)}(x) / \rho_{0}$ and $\bar{\rho}^{(1)}(x) / \rho_{0}$ in Eq. (7), as illustrated for a linear ideal chain in Ref. 51. It is noteworthy that Eqs. (3) and (6) preserve the superior feature of the CABS method, i.e., polymer depletion profiles can be determined from the same set of configuration data for any $x$ and $d$. One only needs a sequence of $\alpha_{i}$ and $\beta_{i}$ data obtained by sampling the configuration space of an unconfined polymer chain, as will be demonstrated in Sec. IV.

In addition to the efficient numerical computations of the depletion profiles, the CABS method has also the theoretical advantage of providing a new physical interpretation of the polymer depletion profiles. One may realize from the Heaviside function in Eq. (3) that $\rho_{i}^{(2)}(x, d) / \rho_{0}$ simply is the joint distribution function of random variables $\alpha_{i}$ and $\beta_{i}$ sampled for an unconfined chain, i.e.,

$$
\frac{\rho_{i}^{(2)}(x, d)}{\rho_{0}}=\operatorname{Pr}\left[\left\{\alpha_{i} \leqslant x\right\} \cap\left\{\beta_{i} \leqslant y\right\}\right] \equiv F_{\alpha_{i}, \beta_{i}}(x, y),
$$

where $y=d-x$, and $\operatorname{Pr}\left[\left\{\alpha_{i} \leqslant x\right\} \cap\left\{\beta_{i} \leqslant y\right\}\right]$ refers to the probability that $\alpha_{i}$ is less than or equal to $x$, and simultaneously $\beta_{i}$ is less than or equal to $y$. In a similar manner, the marginal distribution of $\alpha_{i}$ is obtained from $\rho_{i}^{(1)}(x) / \rho_{0}$ in Eq. (6),

$$
\frac{\rho_{i}^{(1)}(x)}{\rho_{0}}=\operatorname{Pr}\left[\left\{\alpha_{i} \leqslant x\right\} \cap\left\{\beta_{i} \leqslant+\infty\right\}\right] \equiv F_{\alpha_{i}}(x) .
$$

With the assumption of inversion symmetry in the molecular configurations (to be discussed in Sec. III), the two random variables, $\alpha_{i}$ and $\beta_{i}$, are equal in distribution, i.e., $F_{\alpha_{i}}(x)$ $=F_{\beta_{i}}(x)$.

\section{III. $\boldsymbol{R}_{s}$ AND THE DEPLETION LAYER THICKNESS}

We present here properties of the span components, $\alpha_{i}$ and $\beta_{i}$, of a polymer chain in bulk, which are then used in a rigorous proof of Eqs. (1) and (2). As indicated in Fig. 1, the span of a polymer chain with configuration $\{\mathbf{r}\}$ in the $\mathbf{e}_{k}$ direction is divided into two parts by the projection point of bead $i$, i.e., $\alpha_{i}$ and $\beta_{i}$, as defined in Eqs. (4) and (5). In a similar manner, the two span components, $\alpha_{i}$ and $\beta_{i}$, for a polymer chain with configuration $\{-\mathbf{r}\}$ are given by

$$
\begin{aligned}
& \alpha_{i}\left(\{-\mathbf{r}\}, \mathbf{e}_{k}\right)=-\mathbf{r}_{\mathbf{i}} \cdot \mathbf{e}_{k}-\min \left(\{-\mathbf{r}\} \cdot \mathbf{e}_{k}\right), \\
& \beta_{i}\left(\{-\mathbf{r}\}, \mathbf{e}_{k}\right)=\max \left(\{-\mathbf{r}\} \cdot \mathbf{e}_{k}\right)+\mathbf{r}_{\mathbf{i}} \cdot \mathbf{e}_{k} .
\end{aligned}
$$

Since $\max \left(\{-\mathbf{r}\} \cdot \mathbf{e}_{k}\right)=-\min \left(\{\mathbf{r}\} \cdot \mathbf{e}_{k}\right) \quad$ and $\quad\left(\{-\mathbf{r}\} \cdot \mathbf{e}_{k}\right)=\min$ $-\max \left(\{\mathbf{r}\} \cdot \mathbf{e}_{k}\right)$, we have

$$
\begin{aligned}
& \alpha_{i}\left(\{-\mathbf{r}\}, \mathbf{e}_{k}\right)=\beta_{i}\left(\{\mathbf{r}\}, \mathbf{e}_{k}\right), \\
& \beta_{i}\left(\{-\mathbf{r}\}, \mathbf{e}_{k}\right)=\alpha_{i}\left(\{\mathbf{r}\}, \mathbf{e}_{k}\right) .
\end{aligned}
$$

It is understood that the orientation of a polymer chain in free solution without external field is isotropic in space. Therefore, there is an equal probability for the molecule to have configurations $\{\mathbf{r}\}$ and $-\{\mathbf{r}\}$ and, thus, the ensemble averages of $\alpha_{i}$ and $\beta_{i}$ are identical, i.e.,

$$
\left\langle\alpha_{i}\right\rangle=\left\langle\beta_{i}\right\rangle .
$$

In other words, we use the inversion symmetry in the molecular configuration space to derive Eq. (20), and it is worth noting the fact that inversion symmetry is a weaker assumption than isotropic orientation.

The span (maximum projection) $\sigma$ is given by 


$$
\sigma=\alpha_{i}+\beta_{i}=\max \left(\{\mathbf{r}\} \cdot \mathbf{e}_{k}\right)-\min \left(\{\mathbf{r}\} \cdot \mathbf{e}_{k}\right)
$$

for a polymer chain with configuration $\{\mathbf{r}\}$ in the direction of $\mathbf{e}_{k}$. It is evident that although $\alpha_{i}$ and $\beta_{i}$ depend on the position of the $i$ th chain segment, $\sigma$ is certainly independent of the choice of $i$. Furthermore, both $\alpha_{i}$ and $\beta_{i}$ as well as $\sigma$ are translational invariant quantities that depend only on the relative coordinates among monomers. ${ }^{28}$ The ensemble average of $\sigma$ follows Eq. (21),

$$
\langle\sigma\rangle=\left\langle\alpha_{i}\right\rangle+\left\langle\beta_{i}\right\rangle
$$

According to Eqs. (20) and (22), along with the definition of $R_{s}$ being half the average span, it is clear that

$$
R_{s}=\frac{1}{2}\langle\sigma\rangle=\left\langle\alpha_{i}\right\rangle=\left\langle\beta_{i}\right\rangle=\left\langle\alpha_{\mathrm{com}}\right\rangle=\left\langle\beta_{\mathrm{com}}\right\rangle,
$$

which shows that the steric exclusion radius $R_{s}$, introduced previously as half the average span of the molecule, is also identical to the average span with respect to any given segment along the chain as well as the com point in a given direction.

With the identity in Eq. (23) and the relations in Eqs. (6), (7), and (13) for the $i$ th segment, the mean-segment, and the com density distributions, respectively, it is easy to show (by interchanging the order of the integration and the ensemble average) that various definitions of the depletion layer thickness in Eq. (1) give the same value $R_{s}$, i.e.,

$$
\begin{aligned}
& \delta=\int_{0}^{+\infty}\left(1-\frac{\rho_{i}^{(1)}(x)}{\rho_{0}}\right) d x=\left\langle\alpha_{i}\right\rangle=R_{s}, \\
& \delta=\int_{0}^{+\infty}\left(1-\frac{\bar{\rho}^{(1)}(x)}{\rho_{0}}\right) d x=\frac{1}{N} \sum_{i=1}^{N}\left\langle\alpha_{i}\right\rangle=R_{s}, \\
& \delta=\int_{0}^{+\infty}\left(1-\frac{\rho_{\mathrm{com}}^{(1)}(x)}{\rho_{0}}\right) d x=\left\langle\frac{1}{N} \sum_{i=1}^{N} \alpha_{i}\right\rangle=R_{s} .
\end{aligned}
$$

This proves Eqs. (1) and (2). It is important to notice that the proof is solely based on the fact that an inversion symmetry exists in the configuration space of a bulk polymer chain, and no further assumptions have been made on the conformation statistics of the molecule. Equations (1) and (2) are therefore valid not only for polymers under $\Theta$-solvent conditions but also for excluded volume polymers, as well as branched polymers. However, one should bare in mind that the polymer-wall interaction is considered here to be steric exclusion only, under which condition the Boltzmann factor of a hard-wall potential leads to the Heaviside function in the CABS method.

For a slit confinement of width $d$, one may in analogy to Eq. (1) introduce an effective depletion thickness $\Gamma$ in the form

$$
\Gamma=\int_{0}^{d}\left(1-\frac{\rho_{i}^{(2)}(x, d)}{\rho_{0}}\right) d x .
$$

Considering an equilibrium distribution of polymers between

\begin{tabular}{|c|c|c|c|}
\hline & Linear & Ring & Symmetric star \\
\hline$R_{s} / R_{g}$ & $2 / \sqrt{\pi}$ & $\sqrt{\pi / 2}$ & $2 g(f)^{-1 / 2} \psi(f)^{\mathrm{a}}$ \\
\hline
\end{tabular}
a dilute bulk solution phase and the space confined by a slit, one can obtain from $\Gamma$ the total number of polymer chains excluded by the slit confinement as $\rho_{0} A \Gamma$, where $A$ is the area
TABLE I. Characteristic ratios between the steric exclusion radius $R_{s}$ and the root-mean-square radius of gyration $R_{g}$ for ideal chain polymers of linear, ring (Ref. 72), and $f$-arm symmetric star (Ref. 34) architectures.

of the slit plane and $\rho_{0}$ is the uniform polymer concentration (number density) in the bulk solution. The mean polymer concentration in the slit is then obtained by dividing the total number of polymer chains in the slit, $\rho_{0} A(d-\Gamma)$, with the total volume in the slit, $A d$. This leads to a general expression for the equilibrium partition coefficient $K_{0}$ in the form

$$
K_{0}=1-\frac{\Gamma}{d} .
$$

In other words, $\Gamma$ equals the product of the slit width $d$ and the complementary part of the equilibrium partition coefficient, $1-K_{0}$. As is recognized in the literature, ${ }^{80,81}$ the complementary part of the equilibrium partition coefficient derived from Eq. (28) characterizes the selective rejection of solute molecules from a solution by porous membranes in ultrafiltration at the weak flow rate condition. In the weak confinement regime, $K_{0}=1-2 R_{s} / d,^{28,35}$ and therefore, $\Gamma$ $=2 R_{s}$. In the strong confinement regime, $K_{0} \rightarrow 0$, and thus $\Gamma \rightarrow d$.

The identity between $R_{s}$ and the depletion layer thickness $\delta$ is yet another evidence of $R_{s}$ being an important molecular size parameter. In Ref. 28, we showed that $R_{s}$ is the essential molecular size parameter that determines the equilibrium partition partition coefficient $K_{0}$. Furthermore, inspired by the work in Refs. 51, 53, and 57 where $\delta$ was used, we note here that $R_{s}$, because of the identity to $\delta$, may also have a closer relation than, e.g., $R_{g}$ to the classical AsakuraOosawa radius $^{18,19}$ in studies of depletion attraction and to the Navier length in studies of wall slip induced by polymer depletion. ${ }^{57}$ In Table I, the ratios, $R_{s} / R_{g}$, for ideal polymers of different chain architectures are listed. Those constants are characteristics of the polymer architecture.

\section{NUMERICAL RESULTS AND DISCUSSIONS}

Like in the calculations of the equilibrium partition coefficient, $^{28}$ it is straightforward to implement the CABS method for the calculation of polymer depletion profiles as presented in Sec. II. Let us, as an example, present results for linear Gaussian bead-spring polymers.

A Gaussian bead-spring chain is a model polymer molecule represented by a collection of beads linked together with Hookean springs, and it has played a central role in studies of the statistics and dynamics of flexible chain molecules. ${ }^{2}$ The conformational distribution function of such a linear chain of $N$ beads is given by ${ }^{2}$ 

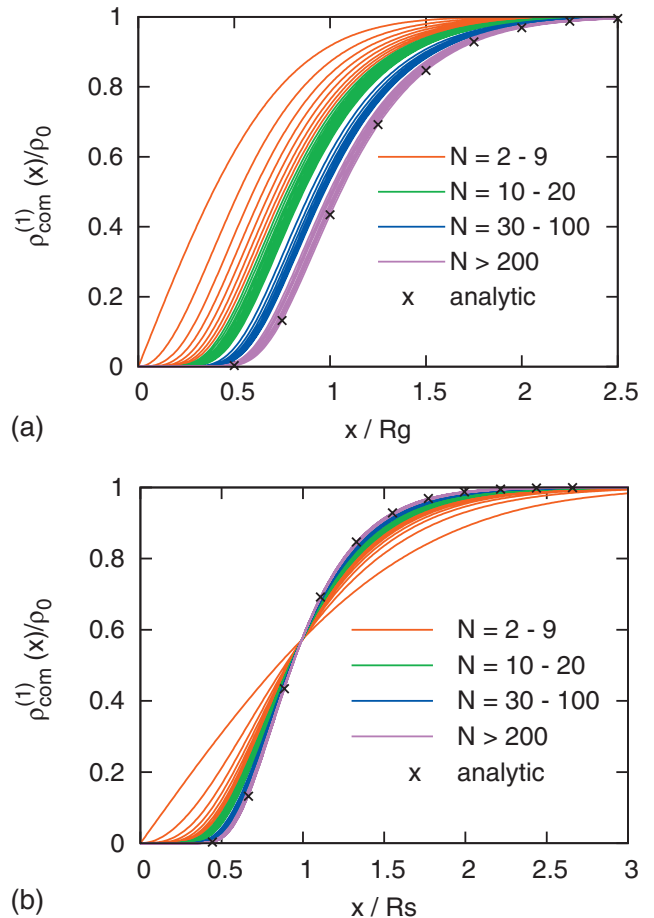

FIG. 2. (Color) Normalized density distribution of the Com near a single wall for linear Gaussian bead-spring chains of $N$ beads. The distance to the wall $x$ is normalized by (a) the radius of gyration $R_{g}$ and (b) the steric exclusion radius $R_{s}$ of the unconfined molecule. The analytical solution by Eisenriegler and Maassen (Ref. 61) is included, which corresponds to $N \rightarrow+\infty$.

$$
\Psi(\{\mathbf{r}\})=\left(\frac{3}{2 \pi b^{2}}\right)^{3(N-1) / 2} \exp \left[-\sum_{i=1}^{N-1} \frac{3\left(\mathbf{r}_{i+1}-\mathbf{r}_{i}\right)^{2}}{2 b^{2}}\right],
$$

where the mean-square bond length $\left\langle\left(\mathbf{r}_{i+1}-\mathbf{r}_{i}\right)^{2}\right\rangle=b^{2}$. In real systems, the conformations of flexible polymers in a melt are often considered to be Gaussian, like the conformation of a flexible polymer dissolved in its $\Theta$-solvent. ${ }^{2}$ In simulations, the Gaussian conformations can be sampled, e.g., by BD simulations of a Rouse chain at equilibrium or by molecular dynamics simulations of polymers in melt state. In this work, polymer conformations are generated by performing random walks of $N-1$ steps with step length following a Gaussian distribution of zero mean and unit variance, i.e., $b^{2}=1$. For each polymer conformation, $\alpha_{i}$ and $\beta_{i}$ are obtained according to Eqs. (4) and (5). Obviously, this can be done for any bead number $i$ as well as the com. The resulting sequences of $\alpha_{i}$ and $\beta_{i}$ data are then used to obtain the depletion profiles. Additionally, we also calculated the root-mean-square radius of gyration $R_{g}$ for comparison with $R_{s}$. The accuracy of the numerical results depends on the total number of chain conformations sampled. For the results reported here, a total of $10^{5}$ configurational snapshots were used for each molecule, and 10 orthonormal vectors of isotropic random orientation were sampled for $\mathbf{e}_{k}$ for each snapshot. Each calculation was repeated ten times to estimate the statistical error. The errors were typically of the order of the line width in all plots and are therefore not shown explicitly.

In Fig. 2, the normalized com density distributions near a single wall for linear Gaussian bead-spring chains of $N$ beads are shown as a function of the distance to the wall, $x$,

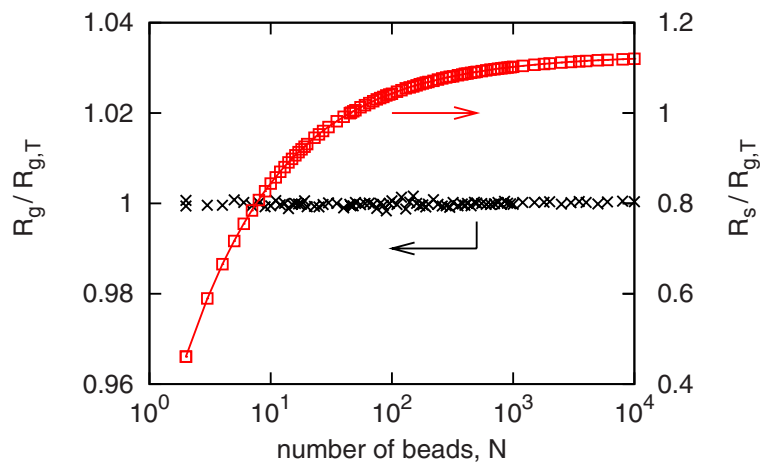

FIG. 3. (Color online) Simulation results of the radius of gyration $R_{g}$ and steric exclusion radius $R_{s}$ with respect to the theoretical values of the radius of gyration $R_{g, T}$, which is predicted by Eq. (30), are plotted as a function of the total number of beads $N$ in a linear Gaussian bead-spring polymer chain. A numerical fit of the $R_{s}$ data is also included.

normalized in Fig. 2(a) by $R_{g}$ and in Fig. 2(b) by $R_{s}$. Both $R_{g}$ and $R_{S}$ are molecular size parameters for the unconfined chain and are obtained from the same set of polymer configurations as those used to calculate the depletion profiles. It is worth mentioning that the depletion profiles of any chain segment can be obtained in a similar effort. We have chosen to use the com representation in order to make a direct comparison with a recent DPD study. ${ }^{47}$ It is noticeable in both figures that as $N$ increases, $\rho_{\text {com }}^{(1)} / \rho_{0}$ will converge to the long chain asymptotic solution. ${ }^{61}$ The use of either $R_{g}$ or $R_{s}$ as the normalization factor gives rise to a distinct feature: in Fig. 2 (a), the depletion profiles persistently shift to the $+x$ direction as $N$ increases, while this is not the case in Fig. 2(b), where all the depletion curves tend to be closer to each other and to the asymptotic behavior. It has been shown recently by Fedosov et al. ${ }^{47}$ that when the depletion thickness $\delta$ was used to normalize the distance to the wall instead of the commonly used $R_{g}$, an almost common curve would appear, just like we find in Fig. 2(b) since $\delta=R_{s}$ in the case of a neutral wall.

To provide an understanding of the background for the different appearances of the curve in Figs. 2(a) and 2(b), we show in Fig. 3 the simulation results of $R_{g}$ and $R_{s}$ as a function of the total number of beads $N$ for a linear Gaussian chain. The theoretical prediction of the root-mean-square radius of gyration [Eq. (11B.1-3) in Ref. 82],

$$
R_{g}=\sqrt{\frac{1}{6} N b^{2}\left(1-N^{-2}\right)},
$$

is used to normalize the results of $R_{g}$ and $R_{s}$ from the simulations. The agreement between the simulation results for $R_{g}$ and the theoretical value in Eq. (30) is excellent, as shown by the horizontal curve at 1.0 in Fig. 3. For an elastic dumbbell $(N=2)$, our simulation yields $R_{g} / b=0.4997 \pm 0.0004$, and Eq. (30) gives $R_{g} / b=1 / 2$. A numerical fit of the form

$$
\begin{aligned}
R_{s} / R_{g}= & (2 / \sqrt{\pi})\left[1-0.7277 N^{-1 / 2}-0.26475 N^{-1}\right. \\
& \left.+0.15781 N^{-3 / 2}\right]
\end{aligned}
$$

is also included and describes well our simulation results of $R_{S}$ (with the maximum deviation less than $0.2 \%$; note that the fit is not based on any physical model). Similar to what we found for linear random walk and self-avoiding walk 

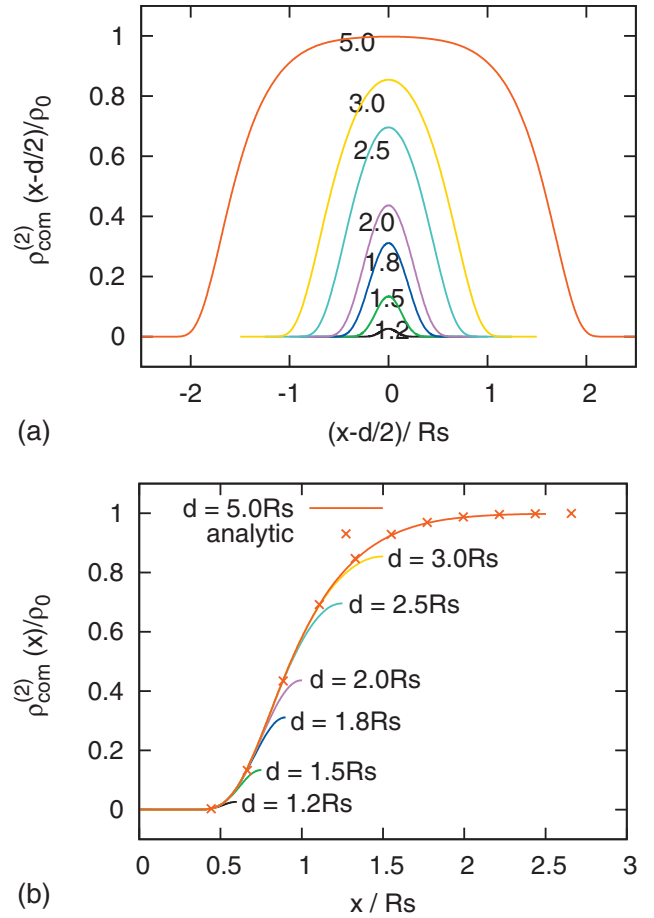

FIG. 4. (Color) Normalized Com density distributions for a linear Gaussian bead-spring polymer of $10^{3}$ beads in a slit confinements of different width. The number inserted for each curve corresponds to the slit width $d$ with respect to $R_{s}$. The axis is chosen such that in (a) the center of the slit is at zero and at (b) the bottom plane of the slit is at zero. The full profile is shown in (a), while only half the profile (from the bottom plane up to the axis of symmetry) is shown in (b). The analytical solution (Ref. 61) corresponds to $N \rightarrow+\infty$ and $d \rightarrow+\infty$.

polymers, ${ }^{28}$ it is evident from Fig. 3 that the ratio $R_{s} / R_{g}$ increases as $N$ increases, and the long chain asymptotic value $R_{S} / R_{g}=2 / \sqrt{\pi}(=1.128379 \cdots)$ is reached only for very large $N$, e.g., we obtain from simulations that $R_{s} / R_{g}=0.9915$ for $N=40$, and $R_{s} / R_{g}=1.1021$ for $N=10^{3}$. From the identity between $\delta$ and $R_{s}$, polymers with the same $R_{s}$ will have the same depletion layer thickness regardless of the total number of beads in the chain. However, for polymers with the same $R_{g}$, the depletion layer thickness will increase as $N$ increases since $R_{s} / R_{g}$ increases with $N$. This accounts for the observation in Fig. 2(a) that the depletion profiles persistently shift to the $+x$ direction as $N$ increases. They have to do so in order to ensure an increase in the depletion layer thickness with respect to $R_{g}$.

The com depletion profiles in slit confining geometries are also calculated as an example for a linear Gaussian beadspring chain of $N=10^{3}$ beads. Results are shown in Fig. 4 for different slit widths $d$. Note that all the results are based on a single sampling of the molecular configuration space. We have chosen the abscissa axis such that the center of slit is at zero in Fig. 4(a), while the bottom plane of the slit is at zero in Fig. 4(b). The full depletion profiles across the slit are shown in Fig. 4(a), while in Fig. 4(b), only the depletion profiles as viewed from the bottom plane up to the axis of symmetry are shown. Clearly, both the com density and its first derivative with respect to $x$ at a distance $(x \rightarrow 0)$ very close to the confining plane are zero, as pointed by Casassa. ${ }^{37}$ For a slit of width $d=5 R_{s}$, our simulation results

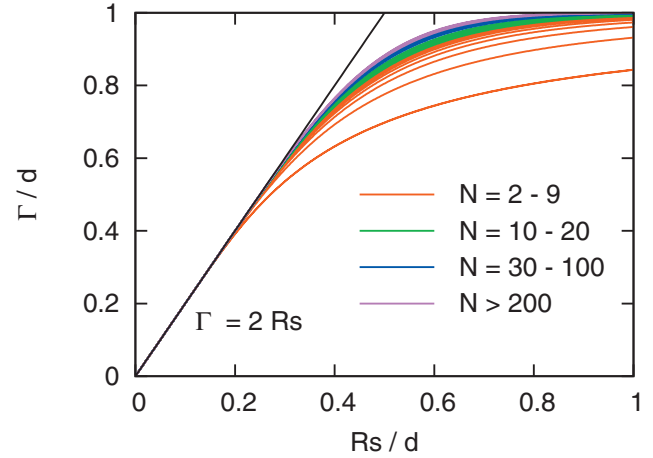

FIG. 5. (Color) Results of $\Gamma / d$ are shown as a function of $R_{S} / d$ for linear Gaussian bead-spring polymers of $N$ beads in a slit confining geometry of width $d$, where $\Gamma$ is the effective depletion thickness introduced in Eq. (28), and $R_{s}$ is the steric exclusion radius of the unconfined polymer chain.

are in a good agreement with the theoretical prediction for a singe wall (i.e., $d=+\infty$ ), as shown in Fig. 4(b). As $d$ decreases, deviation from the analytical solution starts to appear especially near the centerline of the slit, and the deviation is caused by the overlap of the depletion layer due to the other plane. It should be noted that although the CABS approach, in principle, applies for any slit width $d$, in practical calculations, it becomes difficult to determine the depletion profiles accurately in the strong confinement limit such that $d \leqslant R_{s}$. Even though it is possible to improve the statistical accuracy by increasing the total number of sampling, the CABS approach becomes very inefficient since according to Eq. (3) nearly all the $\alpha_{i}$ and $\beta_{i}$ values sampled will yield zero in the calculation.

When the two depletion layers start to overlap, the effective depletion thickness $\Gamma$ depends both on the confinement size and also the molecular details such as the total number of beads in a linear chain. This is shown in Fig. 5, where $\Gamma / d$ is plotted as a function of the molecule-toconfinement size ratio, $R_{s} / d$. In the weak confinement regime (i.e., $R_{s} / d \rightarrow 0$ ), a universal curve is observed, i.e., $\Gamma$ $=2 R_{S}$, corresponding to the fact that each confining plane contributes a depletion layer of thickness $R_{s}$. In the strong confinement regime, $\Gamma$ is less than $2 R_{S}$ since the two depletion layers start to overlap. In this regime and for the same $R_{s} / d$, it is noticeable that $\Gamma / d$ increases with increase in the total number of beads $N$. According to Eq. (28), the equilibrium partition coefficient $K_{0}$ will decrease with the increase of $N$, as we found in Ref. 28.

\section{CONCLUSIONS}

We have demonstrated that the CABS method, originally proposed to calculate the equilibrium partition coefficient of polymers between bulk and confining geometries, ${ }^{28}$ may also be used to determine the entropic depletion profiles of polymers near confining surfaces in dilute solution. We have shown how the depletion profiles can be obtained for a slit of any width especially in the weak confinement regime and for a single wall, and it is straightforward to extend the calculations to include channel and box confining geometries. Furthermore, a new physical interpretation of the polymer depletion profiles arises naturally from the CABS approach, i.e., 
the depletion profile is identical to the cumulative distribution function of the span of an unconfined chain with respect to the chosen reference point in a given direction.

With the only assumption of inversion symmetry in the molecular conformation, we have proven rigorously that in the case of a polymer chain near a wall (i) the depletion layer thickness is the same no matter it is calculated for the com, the mean segment, or the $i$ th segment density distributions, and (ii) the depletion layer thickness equals the steric exclusion radius of the polymer molecule in free space. Both results hold regardless of details in polymer architecture and configuration statistics (such as Gaussian chain or excluded volume chain). We have so far only considered the purely steric exclusion limit where entropic effect dominates, i.e., the confining boundaries are considered to be impenetrable but otherwise passive. However, it is also possible to include surface interactions in the CABS calculation, as will be presented in forthcoming publications.

\section{ACKNOWLEDGMENTS}

Y.W. acknowledges support by the Danish Research Council for Technology and Production Sciences under Grant No. 26-04-0074. G.H.P. acknowledges financial support from the Danish National Research Foundation via a grant to MEMPHYS-Center for Biomembrane Physics. Simulations were performed at the Danish Center for Scientific Computing at the Technical University of Denmark.

${ }^{1}$ P. G. de Gennes, Scaling Concepts in Polymer Physics (Cornell University Press, Ithaca, 1979).

${ }^{2}$ M. Doi and S. F. Edwards, The Theory of Polymer Dynamics (Oxford University Press, New York, 1986).

${ }^{3}$ S. F. Edwards and K. F. Freed, J. Phys. A 2, 145 (1969).

${ }^{4}$ P. G. de Gennes, Rep. Prog. Phys. 32, 187 (1969).

${ }^{5}$ M. Daoud and P. G. de Gennes, J. Phys. (Paris) 38, 85 (1977).

${ }^{6}$ E. Eisenriegler, Polymers Near Surfaces (World Scientific, Singapore, 1993).

${ }^{7}$ E. F. Casassa, J. Polym. Sci., Part B: Polym. Lett. 5, 773 (1967).

${ }^{8}$ J. C. Giddings, E. Kucera, C. P. Russell, and M. N. Myers, J. Phys. Chem. 72, 4397 (1968)

${ }^{9}$ M. E. V. Kreveld, J. Polym. Sci., Polym. Phys. Ed. 13, 2253 (1975).

${ }^{10}$ A. A. Gorbunov, E. B. Zhulina, and A. M. Skvortsov, Polymer 23, 1133 (1982).

${ }^{11}$ M. G. Davidson, U. W. Suter, and W. M. Deen, Macromolecules 20, 1141 (1987)

${ }^{12}$ I. Teraoka, Polymer Solutions: An Introduction to Physical Properties (Wiley, New York, 2002).

${ }^{13}$ K. S. Sorbie, J. Colloid Interface Sci. 139, 299 (1990).

${ }^{14}$ K. S. Sorbie and Y. Huang, J. Colloid Interface Sci. 145, 74 (1991).

${ }^{15}$ P. G. de Gennes, C. R. Acad. Sci. B 288, 359 (1979).

${ }^{16}$ H. D. Hek and A. Vrij, J. Colloid Interface Sci. 84, 409 (1981).

${ }^{17}$ W. B. Russel, D. A. Saville, and W. R. Schowalter, Colloidal Dispersions (Cambridge University Press, Cambridge, 1989).

${ }^{18}$ S. Asakura and F. Oosawa, J. Chem. Phys. 22, 1255 (1954)

${ }^{19}$ S. Asakura and F. Oosawa, J. Polym. Sci. 33, 183 (1958).

${ }^{20}$ J. L. Barrat and J. P. Hausen, Basic Concepts for Simple and Complex Liquids (Cambridge University Press, Cambridge, 2003).

${ }^{21}$ A. Vrij, Pure Appl. Chem. 48, 471 (1976).

${ }^{22}$ E. J. Meijer and D. Frenkel, J. Chem. Phys. 100, 6873 (1994).

${ }^{23}$ W. Poon, Science 304, 830 (2004).

${ }^{24}$ K. E. Eboigbodin, J. R. A. Newton, A. F. Routh, and C. A. Biggs, Langmuir 21, 12315 (2005).

${ }^{25}$ B. Neu and H. J. Meiselman, Biophys. J. 83, 2482 (2002).

${ }^{26}$ D. Marenduzzo and K. Finon, and P. R. Cook, J. Cell Biol. 175, 681
(2006).

${ }^{27}$ P. G. Bolhuis and A. A. Louis, Macromolecules 35, 1860 (2002).

${ }^{28}$ Y. Wang, G. H. Peters, F. Y. Hansen, and O. Hassager, J. Chem. Phys. 128, 124904 (2008).

${ }^{29}$ A. A. Gorbunov and A. M. Skvortsov, Adv. Colloid Interface Sci. 62, 31 (1995).

${ }^{30}$ I. Teraoka, Prog. Polym. Sci. 21, 89 (1996).

${ }^{31}$ R. Tuinier and H. N. W. Lekkerkerker, Macromolecules 35, 3312 (2002).

${ }^{32}$ R. Collins and A. Wragg, J. Phys. A 5, 1196 (1972).

${ }^{33}$ G. K. Stratouras and M. K. Kosmas, Macromolecules 24, 6754 (1991).

${ }^{34}$ E. F. Casassa and Y. Tagami, Macromolecules 2, 14 (1969).

${ }^{35}$ E. F. Casassa, Macromolecules 9, 182 (1976).

${ }^{36}$ E. F. Casassa, Macromolecules 17, 601 (1984).

${ }^{37}$ E. F. Casassa, J. Polym. Sci., Polym. Symp. 72, 151 (1985).

${ }^{38}$ E. F. Casassa, Macromolecules 28, 7756 (1995).

${ }^{39}$ A. T. Clark and M. Lal, J. Chem. Soc., Faraday Trans. 2 77, 981 (1981).

${ }^{40}$ R. Dickman and A. Yethiraj, J. Chem. Phys. 100, 4683 (1994).

${ }^{41}$ H.-P. Hsu and P. Grassberger, J. Chem. Phys. 120, 2034 (2004).

${ }^{42}$ R. M. Jendrejack, D. C. Schwartz, J. J. de Pablo, and M. D. Graham, J. Chem. Phys. 120, 2513 (2004).

${ }^{43}$ J. P. Hernández-Ortiz, H. Ma, J. J. de Pablo, and M. D. Graham, Phys. Fluids 18, 123101 (2006).

${ }^{44}$ O. B. Usta, A. J. C. Ladd, and J. E. Butler, J. Chem. Phys. 122, 094902 (2005).

${ }^{45}$ O. B. Usta, J. E. Butler, and A. J. C. Ladd, Phys. Fluids 18, 031703 (2006).

${ }^{46}$ J. A. Millan, W. Jiang, M. Laradji, and Y. Wang, J. Chem. Phys. 126, 124905 (2007).

${ }^{47}$ D. A. Fedosov, G. E. Karniadakis, and B. Caswell, J. Chem. Phys. 128, 144903 (2008).

${ }^{48}$ F. Rondelez, D. Ausserré, and H. Hervet, Annu. Rev. Phys. Chem. 38, 317 (1987).

${ }^{49}$ D. Kleshchanok, R. Tuinier, and P. R. Lang, J. Phys.: Condens. Matter 20, 073101 (2008).

${ }^{50}$ A. A. Louis, P. G. Bolhuis, J. P. Hansen, and E. J. Meijer, Phys. Rev. Lett. 85, 2522 (2000).

${ }^{51}$ P. G. Bolhuis, A. A. Louis, J. P. Hansen, and E. J. Meijer, J. Chem. Phys. 114, 4296 (2001).

${ }^{52}$ A. A. Louis, P. G. Bolhuis, E. J. Meijer, and J. P. Hansen, J. Chem. Phys. 116, 10547 (2002)

${ }^{53}$ A. A. Louis, P. G. Bolhuis, E. J. Meijer, and J. P. Hansen, J. Chem. Phys. 117, 1893 (2002).

${ }^{54}$ H. Ma and M. D. Graham, Phys. Fluids 17, 083103 (2005).

${ }^{55}$ J. F. Joanny, L. Leibler, and P. G. de Gennes, J. Polym. Sci., Polym. Phys. Ed. 17, 1073 (1979).

${ }^{56}$ G. Allain, C. Ausserre, and F. Rondelez, Phys. Rev. Lett. 49, 1694 (1982).

${ }^{57}$ R. Tuinier and T. Taniguchi, J. Phys.: Condens. Matter 17, L9 (2005).

${ }^{58}$ G. J. Fleer, A. M. Skvortsov, and R. Tuinier, Macromolecules 36, 7857 (2003).

${ }^{59}$ D. A. Svetogorsky, J. Phys. (Paris) 48, 689 (1987).

${ }^{60}$ R. Tuinier, G. A. Vliegenthart, and H. N. W. Lekkerkerker, J. Chem. Phys. 113, 10768 (2000).

${ }^{61}$ E. Eisenriegler and R. Maassen, J. Chem. Phys. 116, 449 (2002).

${ }^{62}$ J. van der Gucht, N. A. M. Besseling, and G. J. Fleer, J. Chem. Phys. 119, 8175 (2003).

${ }^{63}$ J. van der Gucht, N. A. M. Besseling, and G. J. Fleer, Macromolecules 37, 3026 (2004).

${ }^{64}$ E. Eisenriegler and A. Bringer, J. Chem. Phys. 127, 034904 (2007).

${ }^{65}$ W. Feller, Ann. Math. Stat. 22, 427 (1951).

${ }^{66}$ J. J. Weidmann, H. Kuhn, and W. Kuhn, J. Chim. Phys. Phys.-Chim. Biol. 50, 226 (1953).

${ }^{67}$ M. V. Volkenstein, Configurational Statistics of Polymeric Chains (Wiley-Interscience, New York, 1963).

${ }^{68}$ R. J. Rubin, J. Chem. Phys. 56, 5747 (1972).

${ }^{69}$ A. Bellemans, Physica (Amsterdam) 74, 441 (1974).

${ }^{70}$ J. Mazur and R. J. Rubin, J. Chem. Phys. 60, 341 (1974).

${ }^{71}$ R. J. Rubin and J. Mazur, J. Chem. Phys. 63, 5362 (1975).

${ }^{72}$ R. J. Rubin, J. Mazur, and G. H. Weiss, Pure Appl. Chem. 46, 143 (1976).

${ }^{73}$ G. H. Weiss and R. J. Rubin, J. Stat. Phys. 14, 333 (1976).

${ }^{74}$ R. J. Rubin and G. H. Weiss, Macromolecules 10, 332 (1977).

${ }^{75}$ M. Lax and S. Windwer, J. Chem. Phys. 72, 3386 (1980).

${ }^{76}$ Y. A. Makhnovskii, M. E. Maslova, and A. M. Berezhkovskii, Physica A 
225, 221 (1996).

${ }^{77}$ H. E. Daniels, in Proceedings of the Cambridge Philosophical Society, (Cambridge University Press, New York, 1941), Vol. 37, Part 3, pp. 244 251.

${ }^{78}$ H. Kuhn, Experientia 1, 28 (1945).

${ }^{79}$ H. Kuhn, Helv. Chim. Acta 31, 1677 (1948).
${ }^{80}$ T. D. Long and J. L. Anderson, J. Polym. Sci., Polym. Phys. Ed. 22, 1261 (1984).

${ }^{81}$ P. Cifra, T. Bleha, and A. Romanov, Polymer 29, 1664 (1988).

${ }^{82}$ R. B. Bird, C. F. Curtiss, R. C. Armstrong, and O. Hassager, Dynamics of Polymeric Liquids, Kinetic Theory (Wiley, New York, 1986), Vol. 2.

${ }^{83}$ B. H. Zimm and W. H. Stockmayer, J. Chem. Phys. 17, 1301 (1949). 